OPEN ACCESS

Edited by:

Ingo Todt,

Bielefeld University, Germany

Reviewed by:

Sanjeev Mohanty,

Mgm Health Care, India AB Zulkiflee,

University Malaya Medical

Centre, Malaysia

Alexander Bautista,

University of Louisville, United States

*Correspondence:

Jianliang Sun

jxmzsj|@163.com

Specialty section:

This article was submitted to Otorhinolaryngology - Head and Neck Surgery,

a section of the journal

Frontiers in Surgery

Received: 16 March 202

Accepted: 10 August 2021

Published: 08 September 2021

Citation:

Zhang L, Yu Y, Xue J, Lei W, Huang Y,

Li Y and Sun J (2021) Effect of Deliberate Hypotension on Regional

Cerebral Oxygen Saturation During

Functional Endoscopic Sinus Surgery:

A Randomized Controlled Trial.

Front. Surg. 8:681471.

doi: 10.3389/fsurg.2021.681471

\section{Effect of Deliberate Hypotension on Regional Cerebral Oxygen Saturation During Functional Endoscopic Sinus Surgery: A Randomized Controlled Trial}

\author{
Ling Zhang ${ }^{1}$, Yang $\mathrm{Yu}^{1}$, Juan Xue ${ }^{1}$, Weiping Lei ${ }^{2}$, Yaqin Huang ${ }^{2}$, Yong $\mathrm{Li}^{2}$ and \\ Jianliang Sun ${ }^{1 *}$ \\ 1 The Fourth Clinical Medical College, Zhejiang Chinese Medical University School of Medicine, Hangzhou, China, \\ ${ }^{2}$ Department of Anesthesia, Hangzhou First People's Hospital Affiliated to Zhejiang University School of Medicine, Hangzhou, \\ China
}

Background: Deliberate hypotension can reduce bleeding and improve visualization of the surgical field during functional endoscopic sinus surgery (FESS). However, hypotension may cause brain hypoperfusion and subsequent ischemic injuries, such as delayed awakening, stroke, postoperative delirium, and postoperative cognitive dysfunction. Near-infrared spectroscopy (NIRS) can be used to monitor real-time regional cerebral oxygen saturation $\left(\mathrm{rSO}_{2}\right)$ levels to estimate brain perfusion. The present study aimed to evaluate the change in $\mathrm{rSO}_{2}$ induced by deliberate hypotension during FESS, and assess the impact of deliberate hypotension on the surgical process.

Material and Methods: A randomized controlled trial was registered with the Chinese clinical trial registry (ChiCTR2000039846). A total of 40 patients were enrolled and randomly divided into the control and intervention groups, and finally, 39 patients were analyzed. Deliberate hypotension was induced in the intervention group using nicardipine and esmolol, whereas the control group received general anesthesia without deliberate hypotension. We recorded mean arterial pressure (MAP), saturation of pulse oximetry $\left(\mathrm{SpO}_{2}\right), \mathrm{rSO}_{2}$, and heart rate $(\mathrm{HR})$ before induction of anesthesia (T0), immediately after induction of anesthesia (T1), at the beginning of the operation (corresponding with the establishment of deliberate hypotension) (T2), $10 \mathrm{~min}$ (T3) and $20 \mathrm{~min}$ (T4) after the operation began, at the end of the operation (corresponding with the end of deliberate hypotension) (T5), and $5 \mathrm{~min}$ (T6) and $15 \mathrm{~min}$ (T7) after the operation. The partial pressure of end-tidal carbon dioxide $\left(\mathrm{PetCO}_{2}\right)$ was recorded at T1, T2, T3, T4, T5, and T6. The duration of surgery, intraoperative blood loss, tracheal extubation time, and the number of patients that experienced cerebral desaturation events (CDEs) were recorded. The surgical field was estimated postoperation based on the Fromme score.

Results: A 30\% decrease from the baseline MAP resulted in a decrease of intraoperative bleeding, improvement in the quality of the surgical field, and the shortening of the duration of surgery during FESS in the intervention 
group compared with the control group. In addition, $\mathrm{rSO}_{2}$ was reduced and no CDEs were experienced in the intervention group. Linear regression analysis demonstrated a correlation between the decline in $\mathrm{rSO}_{2}$ and that in MAP.

Conclusions: A decrease in MAP to a certain level will cause a decrease of $\mathrm{rSO}_{2}$ in patients undergoing FESS under general anesthesia. Based on our findings, we recommend that the deliberate hypotensive target indicated by MAP be reduced by $30 \%$, while $\mathrm{PetCO}_{2}$ is maintained at $35-40 \mathrm{mmHg}$ and $\mathrm{HR}$ is maintained at about 60 beats per minute during FESS.

Keywords: functional endoscopic sinus surgery, deliberate hypotension, regional cerebral oxygen saturation, mean arterial pressure, nicardipine, esmolol

\section{INTRODUCTION}

With the development of nasal visualization technology, functional endoscopic sinus surgery (FESS) has become the gold standard for treating medically refractory chronic sinusitis. Given the sensitive anatomical structures in the narrow spaces of the nasal cavities, such as the carotid artery, anterior ethmoidal artery, orbit, optic nerve, and intracranial contents of the anterior cranial fossa just beyond the skull base, even a small visible bleed into the surgical field may affect the surgical field of vision, thereby causing tissue damage and serious complications $(1,2)$. Thus, an optimal surgical field of view is crucial for adequate visualization and identification of sensitive neurovascular structures (2).

At present, various pre- and intraoperative strategies can be used to reduce or prevent bleeding into the surgical field and improve visualization, such as the decreasing of the mean arterial pressure (MAP), lowering heart rate (HR), local injection of vasoconstrictors, application of topical sinonasal vasoconstrictors, preoperative use of steroids, reversal of the Trendelenburg position, and induction of deliberate hypotension with anesthesia (3). Deliberate hypotension is defined as the reduction of systolic blood pressure (BP) to $80-90 \mathrm{mmHg}$, and that of MAP to $50-65 \mathrm{mmHg}$ or $30 \%$ lower than the baseline level (4). However, extreme hypotension may cause organ insufficiency and subsequent ischemic injury of vital organs, especially the brain (1).

Near-infrared spectroscopy (NIRS) is a non-invasive method of monitoring regional cerebral oxygen saturation $\left(\mathrm{rSO}_{2}\right)$ in real time (5) that allows estimation of brain perfusion and early detection of cerebral desaturation events (CDEs), which is defined as a $>20 \%$ reduction in $\mathrm{rSO}_{2}$ for at least $15 \mathrm{~s}$ or $<55 \%$ drop in its absolute level (5). NIRS can diagnose insufficient oxygen saturation on time, and prevent hypoxemia and other complications of anesthesia (6). Cerebral oximetry was first used for intraoperative monitoring during cardiac surgery to evaluate cerebral perfusion (7). It has been gradually adopted in non-cardiac operations as well, such as thoracic surgery and vascular surgery (7). Recent reports suggest that NIRS can guide deliberate hypotension by monitoring cerebral perfusion during FESS $(2,8)$. However, these studies are only prospective observational studies.
The present study aimed to conduct a randomized controlled trial (RCT) to evaluate the change in $\mathrm{rSO}_{2}$ induced by deliberate hypotension during FESS, and assess the impact of deliberate hypotension on surgery.

\section{MATERIALS AND METHODS}

\section{Patients}

The RCT was conducted at the Hangzhou First People's Hospital affiliated with Zhejiang University School of Medicine following approval by the Ethics Committee (2020YLSD00201 ) and was registered with the Chinese clinical trial registry (ChiCTR2000039846). A total of 40 patients who were diagnosed with chronic rhinosinusitis and scheduled for FESS were enrolled in this study and all participants signed the informed consent form. The inclusion criteria were as follows: (1) patients with an American Society of Anesthesiologists (ASA) class I and II status; (2) basal BP $\leq 140 / 90 \mathrm{mmHg}$; and (3) BP of hypertensive patients with antihypertensive agents $\leq 140 / 90 \mathrm{mmHg}$. The exclusion criteria were as follows: (1) hemoglobin (Hb) levels $<100 \mathrm{~g} / \mathrm{L}$; (2) preoperative mini-mental state examination (MMSE) score $\leq 23$ points; (3) cerebrovascular disease; (4) anxiety and mental disorders; (5) uncontrolled hypertension; and (6) coagulopathy and use of platelet inhibitors or anticoagulant therapy.

The patients were randomly assigned to the control and intervention groups ( $n=20$ each) using a list of numbers generated by the QuickCalcs (GraphpadPrism 7). The group assignment numbers were sealed in an envelope, and opened once written informed consent was obtained. The groups were homogenous in terms of age, gender, weight, $\mathrm{Hb}$ levels, baseline $\mathrm{MAP}$ and $\mathrm{rSO}_{2}$, and preoperative MMSE scores.

\section{Experimental Protocols}

The patients were instructed to fast before the operation as per standard guidelines. ECG, HR, non-invasive BP, saturation of pulse oximetry $\left(\mathrm{SpO}_{2}\right)$, temperature, and bispectral index (BIS) were measured in the operating room with the patient in supine position. Then, the right radial artery was punctured and catheterized to monitor invasive arterial BP. NIRS sensors (FORE-SIGHT oximeter, CASMED) were placed on both the right and left sides of the forehead before the induction of anesthesia to monitor $\mathrm{rSO}_{2}$. The baseline right and left 
$\mathrm{rSO}_{2}$ were measured before anesthesia induction when the patient breathed the room air, and the mean was calculated. Midazolam $(0.04 \mathrm{mg} / \mathrm{kg})$ was injected $5 \mathrm{~min}$ before the induction of anesthesia, and the baseline MAP was recorded 5 min later. Anesthesia was induced with propofol $(1 \mathrm{mg} / \mathrm{kg})$, etomidate (0.2 $\mathrm{mg} / \mathrm{kg})$, sufentanil $(0.5 \mu \mathrm{g} / \mathrm{kg})$, and cisatracurium $(0.2$ $\mathrm{mg} / \mathrm{kg}$ ), and tracheal intubation was performed. Volumecontrolled ventilation was provided with tidal volume 6-8 $\mathrm{ml} / \mathrm{kg}$, the fraction of inspired oxygen maintained at $50 \%$ in an oxygen air mixture, with a respiration rate $12-15$ times per minute. The respiration rate and tidal volume were adjusted according to the partial pressure of endtidal carbon dioxide $\left(\mathrm{PetCO}_{2}\right)$ of $35-40 \mathrm{mmHg}$. BIS was maintained between 40 and 60 with a continuous infusion of propofol and remifentanil, and continuous inhalation of $1-1.5 \%$ sevoflurane. A total of $2 \mathrm{ml}$ lignocaine-adrenaline $(1: 2,00,000)$ was injected into the operative region at the beginning of the operation.

Before surgery, hypotension was induced immediately by reducing MAP to $70 \%$ of the baseline and was maintained by continuous infusion of nicardipine plus esmolol in the intervention group (nicardipine $20 \mathrm{mg}$ plus esmolol $200 \mathrm{mg}$ diluted $0.9 \%$ saline solution to $50 \mathrm{ml}$ and adjusting infusion rate by MAP level). The control group received only general anesthesia without deliberate hypotension. In case CDEs were encountered, nicardipine and esmolol infusion was stopped, anesthetic drugs were adjusted, and the patients were given fluid therapy and $5 \mathrm{mg}$ intravenous ephedrine if needed.

At the ending of the operation, all drugs were discontinued. The tracheal catheter was removed when the patient regained spontaneous breathing and consciousness, recovered cough and swallowing reflex, the patient could lift his/her head off the pillow for more than $5 \mathrm{~s}$, the breathing rate was 10-20 times/min and $\mathrm{SpO}_{2}>95 \%$ with breathing air.

Mean arterial pressure, $\mathrm{SpO}_{2}, \mathrm{HR}$, and $\mathrm{rSO}_{2}$ were recorded before induction of anesthesia (T0), immediately after induction of anesthesia (T1), at the beginning of the operation (which corresponded to the establishment of deliberate hypotension) (T2), $10 \mathrm{~min}$ (T3) and $20 \mathrm{~min}$ (T4) after the operation began, at the end of the operation (which corresponded to the end of deliberate hypotension) (T5), and finally $5 \mathrm{~min}$ (T6) and $15 \mathrm{~min}$ (T7) after the end of the operation. PetCO $\mathrm{O}_{2}$ was recorded at T1, T2, T3, T4, T5, and T6. The duration of surgery (the duration of deliberate hypotension), intraoperative blood loss, tracheal extubation time, and the number of patients that experienced CDEs were recorded.

After the surgery, the surgical field was evaluated by the surgeon using Fromme score, which is as follows: 1-slight bleeding without the requirement for blood suctioning, 2-slight bleeding requiring occasional suctioning but surgical field not compromised, 3-slight bleeding requiring frequent suctioning and surgical field affected within a few seconds of removing suction, 4-moderate bleeding requiring frequent suctioning and surgical field directly compromised by bleeding after suction is removed, and 5-severe bleeding that overwhelmed constant suctioning and severely compromised the surgical field and prevented surgery (9).
Cognitive function was tested on the evening before surgery was performed and 1 day after surgery using the MMSE scale.

\section{The INVOS System}

FORE-SIGHT oximeter (CASMED) is a two-channel probe that allows continuous, non-invasive, and real-time measurement of cerebral oxygenation. The FORE-SIGHT oximeter is a two-channel (right and left) NIRS cerebral oximeter that automatically analyzes and calculates the $\mathrm{rSO}_{2}$ value detected by sensors.

\section{Statistical Analysis}

The sample size for calculating the difference in $\mathrm{rSO}_{2}$ between the two groups during deliberate hypotension was determined using G-power (version 3.1.9.2, $\alpha=0.05$, power $=0.8$ ) depending on our preliminary study results (intervention group: mean $=$ $70.83, \mathrm{SD}=3.041$; control group: mean $=73.5, \mathrm{SD}=2.437$, effect size $=0.969$ ). A total of 18 patients were required per group, and considering an expulsion rate of 0.2 , the sample size was increased to 20 patients per group.

All variables were expressed as numbers, mean (SD) or median (interquartile range). Statistical analysis was performed using the GraphPad Software (Prism7 for Mac OSX). Normally distributed continuous data were compared using the unpaired $t$-test, and the Mann-Whitney $U$-test was used for data with non-normal distribution. Two-way ANOVA was used to analyze serial changes in $\mathrm{HR}, \mathrm{MAP}, \mathrm{PetCO}_{2}$, and $\mathrm{rSO}_{2}$ data with time using the intragroup and group values as interfactor measurements. Spearman's correlation analysis was used to analyze the relationship between percentage decrease in $\mathrm{rSO}_{2}$ and MAP. Categorical variables were analyzed using Pearson's chi-square test or Fisher's exact test. $P<0.05$ was considered to indicate statistical significance.

\section{RESULTS}

Based on the inclusion and exclusion criteria, 40 patients were initially enrolled in this study, and one patient was later excluded due to loss of data (Figure 1). The demographic data of the remaining 39 patients are shown in Table 1, which indicates a lack of any significant difference between the control and intervention groups in terms of age, gender, weight, $\mathrm{Hb}$ levels, history of hypertension, and ASA physical status $(P>0.05)$. Table 2 shows the results of the variables monitored during surgery. Duration of surgery, Fromme score, and intraoperative blood loss in the intervention group were lower than that of the control group $(P<0.05)$. However, the tracheal extubation time in the intervention group was longer, compared with the control group $(P<0.05)$. The changes in MAP during FESS are shown in Table 3; Figure 2A. There was no difference in the baseline MAP values at T0 $(P>0.05)$. The MAP values at T1, T2, T3, T4, T5, and T6 were lower than that at T0 (baseline) in both the control and intervention groups $(P<0.05)$. However, the MAP values were restored to the baseline level at T7 $(P>0.05)$. Compared with the control group, the MAP values were lower in the intervention group at T2, T3, T4, and T5 $(P<0.05)$. 


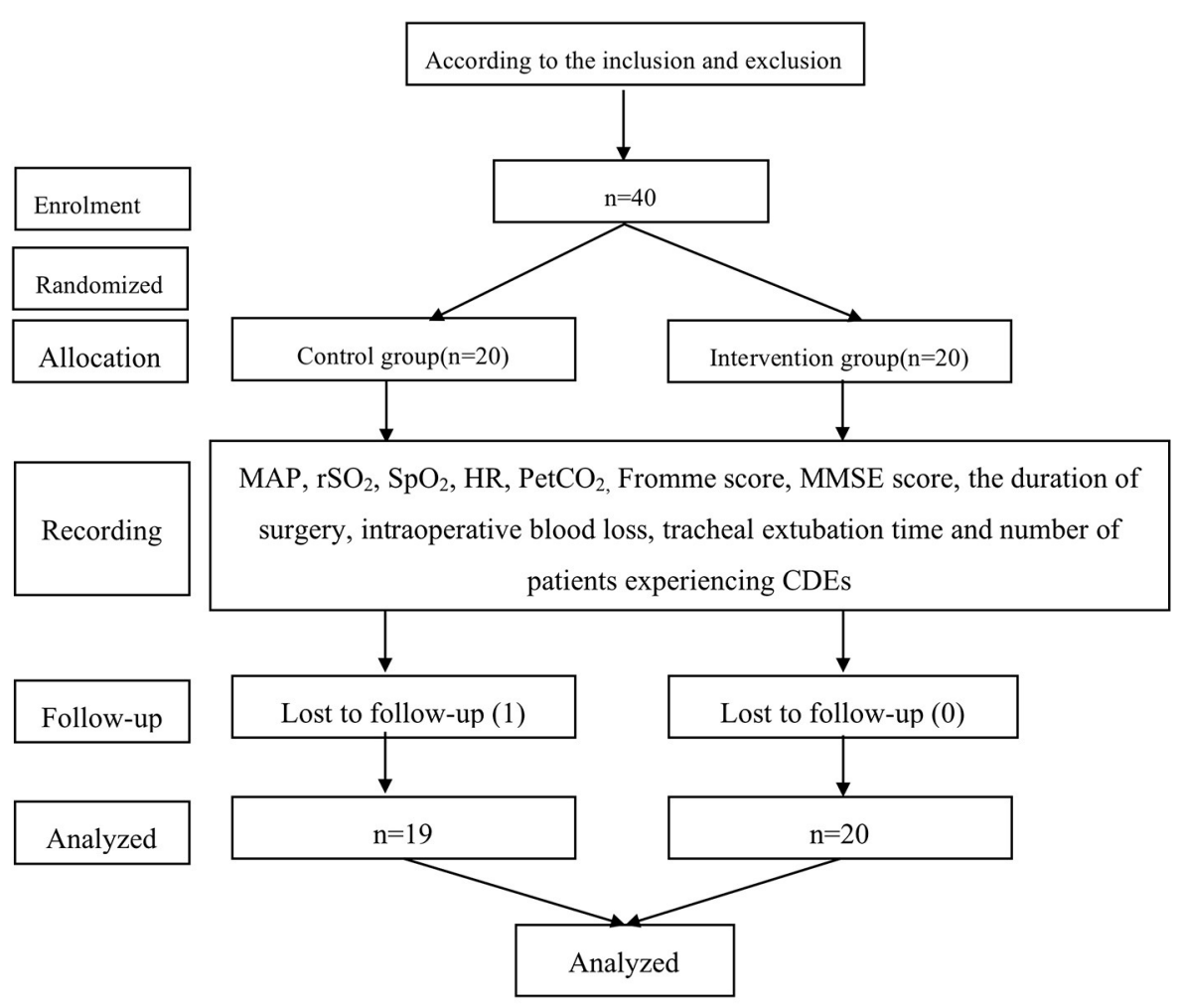

FIGURE 1 | Study flowchart.

TABLE 1 | Demographic and preoperative characteristics.

\begin{tabular}{lccc}
\hline Variable & Control $(\boldsymbol{n}=\mathbf{1 9})$ & Intervention $(\boldsymbol{n}=\mathbf{2 0})$ & $\boldsymbol{P}$-value \\
\hline Age (years) & $48 \pm 13.86$ & $50.55 \pm 14.34$ & 0.576 \\
Gender (male/female) & $13 / 6$ & $12 / 8$ & 0.741 \\
ASA physical status (I/II) & $1 / 18$ & $3 / 17$ & 0.605 \\
Weight (kg) & $66 \pm 10.86$ & $63.1 \pm 10.59$ & 0.404 \\
Hypertension, $n$ & 5 & 4 & 0.716 \\
Hemoglobin $(\mathrm{g} / \mathrm{l})$ & $146.50 \pm 13.71$ & $143.10 \pm 16.76$ & 0.491 \\
\hline
\end{tabular}

All values are given as mean $\pm S D$ or number of patients. ASA, American Society of Anesthesiologists.

Changes in $\mathrm{rSO}_{2}$ are shown in Table 4; Figure 2B during FESS. There was no difference in the baseline $\mathrm{rSO}_{2}$ values at T0 $(P>0.05)$ and the baseline $\mathrm{rSO}_{2}$ value was $75.89 \%$ in our study. The $\mathrm{rSO}_{2}$ values at T2, T3, T4, T5, and T6 were lower than that at T0 (baseline) in the intervention group ( $P$ $<0.05)$, while the value was restored to the baseline level at T7 $(P>0.05)$. Unlike the pattern of the MAP values, there was no additional decrease in $\mathrm{rSO}_{2}$ values in the control group $(P>0.05)$. In both groups, the $\mathrm{rSO}_{2}$ values at $\mathrm{T} 1$ were not lower than that at T0 $(P>0.05)$. Compared with the control group, the $\mathrm{rSO}_{2}$ values were significantly lower in the intervention group at T2, T3, T4, T5, and T6 $(P<$ $0.05)$. However, there was no significant difference at $\mathrm{T} 1$ and T7 $(P>0.05)$.
TABLE 2 | Clinical characteristics of control and intervention groups.

\begin{tabular}{lccc}
\hline Variable & Control $(\boldsymbol{n}=\mathbf{1 9})$ & Intervention $(\boldsymbol{n}=\mathbf{2 0})$ & $\boldsymbol{P}$-value \\
\hline $\begin{array}{l}\text { Duration of surgery } \\
\text { (min) }\end{array}$ & $93.11 \pm 33$ & $71.85 \pm 29.36^{*}$ & 0.040 \\
$\begin{array}{l}\text { The tracheal } \\
\text { extubation time (min) }\end{array}$ & $8.79 \pm 3.54$ & $11.80 \pm 4.94^{*}$ & 0.036 \\
$\begin{array}{l}\text { Fromme score } \\
\text { Intraoperative blood }\end{array}$ & $3.37 \pm 0.60$ & $2.80 \pm 0.52^{* \star}$ & 0.003 \\
loss (mL) & $77(55-100)$ & $47(30-101.3)^{*}$ & 0.034 \\
& & & \\
\hline
\end{tabular}

All values are given as mean $\pm S D$ or median (interquartile range). ${ }^{\star} P<0.05,{ }^{\star \star} P<0.01$.

The decline in cognitive function on day 1 postoperative was measured using MMSE but was not observed in any of the patients $(P>0.05)$ (Table 5). Figures $2 \mathrm{C}-\mathbf{E}$ shows that the intraoperative parameters, $\mathrm{HR}, \mathrm{PetCO}_{2}$, and $\mathrm{SpO}_{2}$, at each recording point were similar between these groups $(P>0.05)$. Figure 3 shows the decreases in MAP and $\mathrm{rSO}_{2}$ from the baseline in the deliberate hypotension group $(P<0.05)$. When MAP decreased by $\sim 30 \%$, the percentage decrease of $\mathrm{rSO}_{2}$ from the baseline was $\sim 5 \%$ in the intervention group.

Analysis of all patients, irrespective of the group they belonged to, demonstrated a significant correlation between the percentage decrease in MAP and the percentage decrease in $\mathrm{rSO}_{2}(r=0.627$, 95\% CI: 0.546-0.695). 
TABLE 3 | Comparison of MAP $(\mathrm{mmHg})$ at each time point between control and intervention groups.

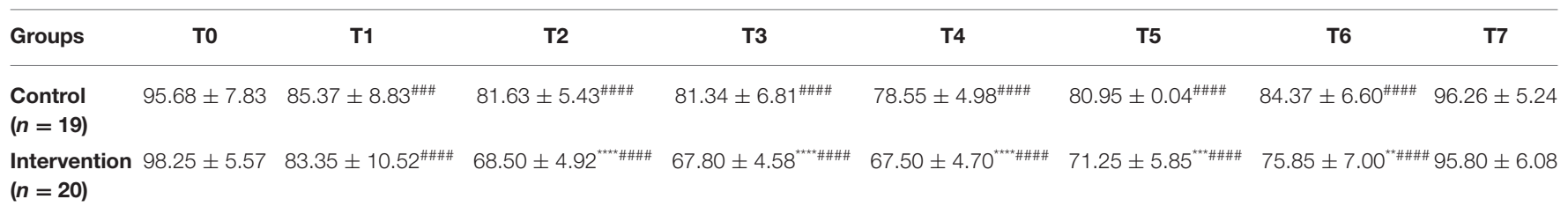

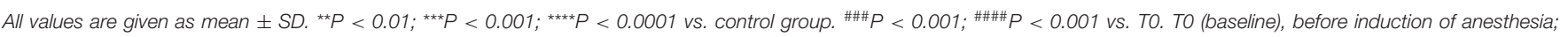

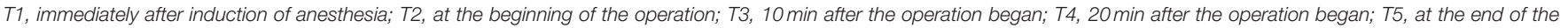
operation; T6, $5 \mathrm{~min}$ after the end of the operation; T5, $15 \mathrm{~min}$ after the end of the operation. MAP, mean arterial pressure.

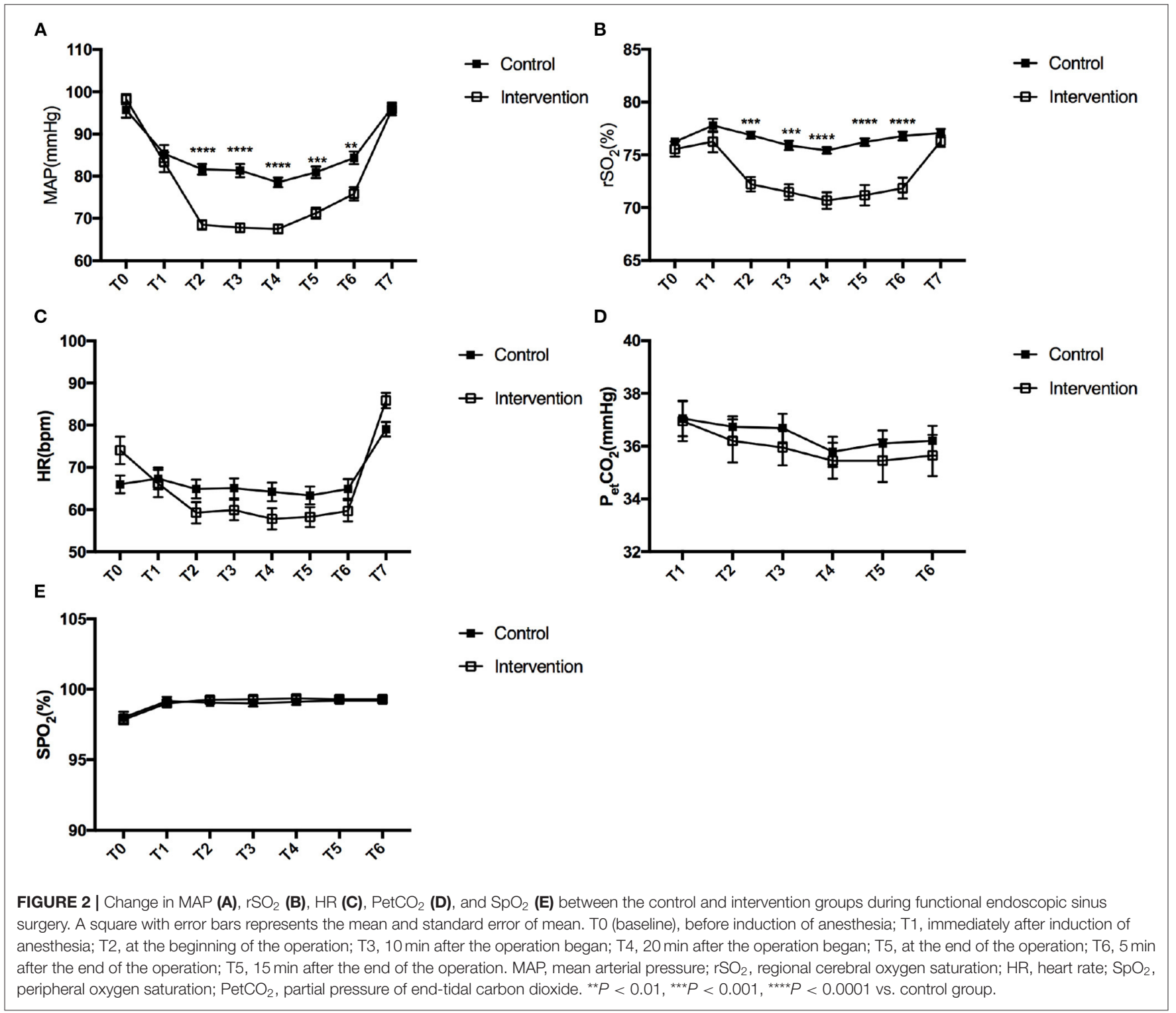

\section{DISCUSSION}

In this study, we observed that a $30 \%$ decrease of baseline MAP resulted in decreased intraoperative bleeding, improved quality of the surgical field, and resulted in a shorter duration of surgery during FESS. More importantly, although $\mathrm{rSO}_{2}$ values decreased, none of the patients experienced CDEs. Correlation analysis of the percentage of decrease of $\mathrm{rSO}_{2}$ and MAP demonstrated a correlation between the decline of $\mathrm{rSO}_{2}$ and the level of decrease of MAP. At the same time, analysis of cognitive function 
TABLE 4 | Comparison of $\mathrm{rSO}_{2}(\%)$ at each time point between control and intervention groups.

\begin{tabular}{|c|c|c|c|c|c|c|c|c|}
\hline Groups & TO & T1 & T2 & T3 & T4 & T5 & T6 & T7 \\
\hline $\begin{array}{l}\text { Control } \\
(n=19)\end{array}$ & $76.24 \pm 1.50$ & $77.79 \pm 2.73$ & $76.87 \pm 1.49$ & $75.90 \pm 1.88$ & $75.42 \pm 1.33$ & $76.21 \pm 1.55$ & $76.79 \pm 1.84$ & $77.08 \pm 1.69$ \\
\hline $\begin{array}{l}\text { Intervention } \\
(n=20)\end{array}$ & $75.55 \pm 3.13$ & $76.25 \pm 4.54$ & $72.23 \pm 3.04^{\star \star \star \#}$ & $71.48 \pm 3.28^{\star \star \star \# \# \#}$ & 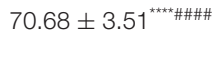 & $71.18 \pm 4.34^{\star \star \star \star \# \# \# ~}$ & $71.85 \pm 4.45^{\star \star \star \star \star \# \# ~}$ & $76.3 \pm 2.45$ \\
\hline
\end{tabular}

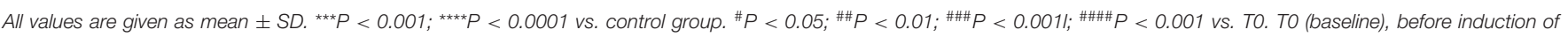

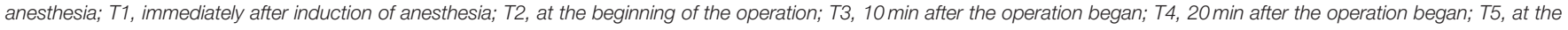
end of the operation; T6, $5 \mathrm{~min}$ after the end of the operation; T5, $15 \mathrm{~min}$ after the end of the operation. $\mathrm{rSO}_{2}$, regional cerebral oxygen saturation.

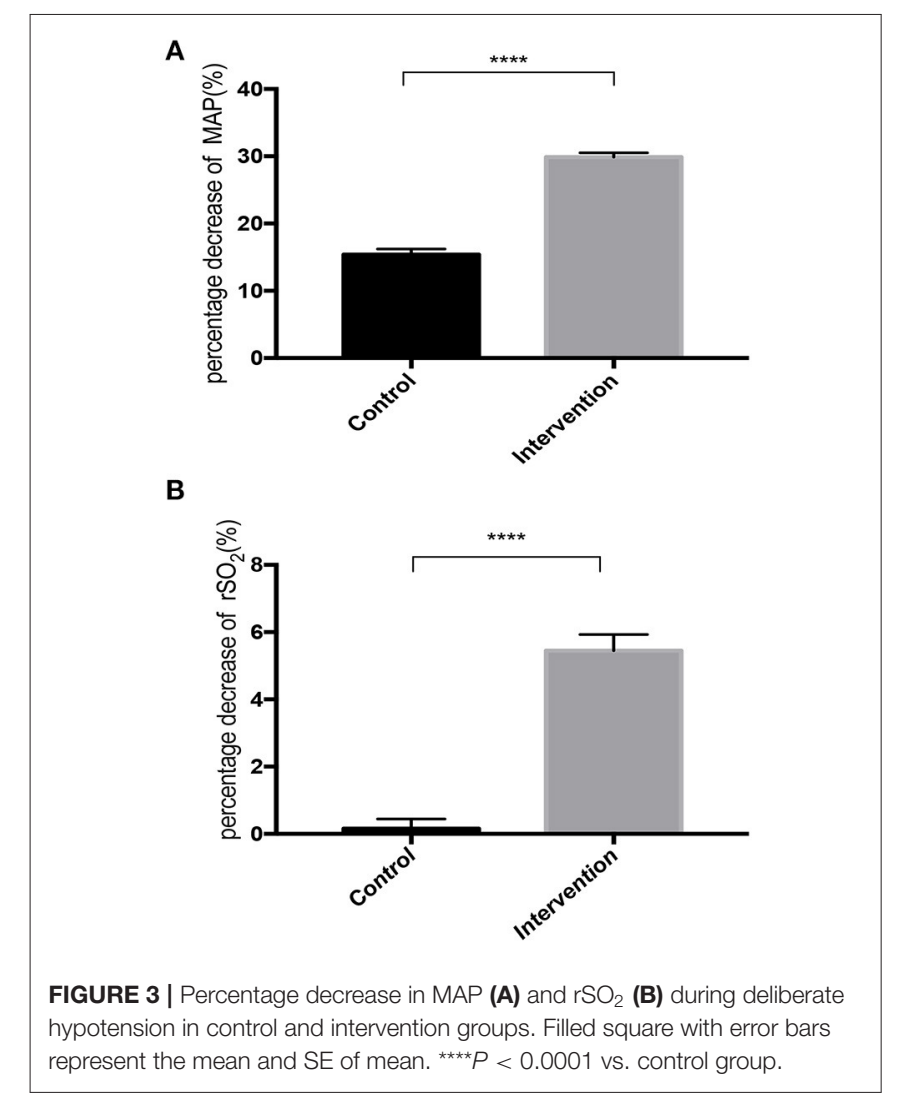

TABLE 5 | Comparison of preoperative and postoperative MMSE scores.

\begin{tabular}{lccc}
\hline & Control $(\boldsymbol{n}=\mathbf{1 9})$ & Intervention $(\boldsymbol{n}=\mathbf{2 0})$ & $\boldsymbol{P}$-value \\
\hline Preoperative & $28.58 \pm 0.86$ & $28.85 \pm 0.99$ & 0.873 \\
Postoperative first day & $28.73 \pm 0.93$ & $28.50 \pm 1.10$ & 0.885 \\
\hline
\end{tabular}

Data are presented as mean $\pm S D$. MMSE, mini-mental state examination.

on day 1 postoperation showed that postoperative cognitive dysfunction (POCD) caused by MMSE was not observed in any of the patients.

Effective hemostasis is crucial during FESS since even minor bleeding can severely compromise an already restricted view in the narrow nasal space, prolong the operation, and increase the risk of damage to sensitive tissue structures. The lowering of MAP during general anesthesia can minimize intraoperative bleeding, reduce the time of operation, and improve the quality of surgery $(3,10)$. A prospective, observational cohort study showed that MAP below $60 \mathrm{mmHg}$ was associated with better surgical visibility in terms of bleeding assessment score (1). Consistent with this, we found that the quality of surgical field was enhanced in the intervention group compared to the control group in terms of Fromme score, which indicates less bleeding and a shorter duration of surgery.

However, a lower MAP can decrease cerebral blood flow $(\mathrm{CBF})$ and thus increase the risk of cerebral hypoperfusion, eventually leading to postoperative neurological injuries, such as stroke, postoperative delirium (POD), and POCD (11). A large retrospective cohort analysis of 7,457 patients undergoing cardiac surgery showed a strong association between continuous MAP below $64 \mathrm{mmHg}$ and postoperative stroke during cardiopulmonary bypass, and the severity and duration of hypotension were closely connected with the risk of stroke after surgery (12). Another case-control study of non-cardiac and non-neurosurgical procedures showed that the duration of baseline MAP reduction by $30 \%$ was linked with stroke within 10 days postsurgery (13). Similarly, a retrospective study conducted on 1,083 patients after general anesthesia showed that $35 \%$ of patients suffered from delirium after surgery, and perioperative hypotension was moderately correlated with higher odds of postoperative delirium (14).

Cerebral pressure autoregulation, MAP between 60 and 150 $\mathrm{mmHg}$, maintains a relatively stable CBF. However, cerebral autoregulation is subject to variation, and its plateau, lower and upper limits are affected by age, gender, diseases, metabolic rate, vasoactive drugs, volatile anesthetics, sympathetic tone, $\mathrm{Hb}$ and oxygen content, and $\mathrm{CO}_{2}$ levels (15). Continuous monitoring of $\mathrm{rSO}_{2}$ using the NIRS can reflect the oxygen supply, oxygen consumption, and cerebral perfusion on time (16). Studies have shown that decreased intraoperative $\mathrm{rSO}_{2}$ is significantly associated with postoperative neurological dysfunction (17-19). A prospective cohort study including 43 patients undergoing cerebral endovascular surgery showed that the decrease of $\mathrm{rSO}_{2}$ can be used to predict delirium and that the continuous monitoring of $\mathrm{rSO}_{2}$ can reduce the incidence of postoperative delirium (19). Another study revealed that the duration of decline in $\mathrm{rSO}_{2}<60 \%$ during lumbar spinal surgery was correlated with the development of POCD (17). Therefore, monitoring of $\mathrm{rSO}_{2}$ and timely intervention can reduce the occurrence of postoperative neurological disorders. The criteria for cerebral 
ischemia are reduction of 10 index points in $\mathrm{rSO}_{2}$ from a stable baseline, absolute value of $\mathrm{rSO}_{2}<50 \%, 20-25 \%$ reduction in relative $\mathrm{rSO}_{2}$, and $>25 \%$ difference between the left and right side $\mathrm{rSO}_{2}$ (7). Thus, a $20 \%$ drop in $\mathrm{rSO}_{2}$ from the baseline was used as the threshold in this study, and deliberate hypotension was stopped in the event of further drop till $\mathrm{rSO}_{2}$ was restored to acceptable levels.

We surveyed the effect of controlled hypotension on $\mathrm{rSO}_{2}$ during FESS. Our results indicated a correlation between the decline in $\mathrm{rSO}_{2}$ and the decrease in MAP $(r=0.627, P<$ 0.0001). An observational study of 41 patients undergoing FESS found that MAP was moderately cross-correlated with current $\mathrm{rSO}_{2}(r=0.728)(2)$. At the same time, the correlation analysis between $\mathrm{PetCO}_{2}$ and $\mathrm{rSO}_{2}$ also found a correlation between them (2). However, a prospective, blinded, observational trial conducted on 31 healthy patients showed that cerebral oximetry was directly correlated with $\mathrm{PetCO}_{2}$ but not with MAP during sinus endoscopy (8). $\mathrm{CO}_{2}$ levels can influence $\mathrm{CBF}$ by regulating the dilation and constriction of cerebral vessels and alter $\mathrm{rSO}_{2}$ levels (20). Therefore, we kept $\mathrm{PetCO}_{2}$ between the control and intervention groups at a similar level. In our study, carbon dioxide was found to be an essential factor that affected $\mathrm{rSO}_{2}$ control. Thus, our results show that a reduction in $\mathrm{rSO}_{2}$ is related to the level of decrease in MAP. Previously published results have shown that the $\mathrm{rSO}_{2}$ value decreased when patients were placed in the beach-chair position, then induced hypotension did not lead to a reduction of the $\mathrm{rSO}_{2}$ value while undergoing arthroscopic shoulder surgery (21). Therefore, when other major factors that affect $\mathrm{rSO}_{2}$ are controlled, the $\mathrm{rSO}_{2}$ value is directly correlated with MAP.

Our results showed that there was no difference between baseline MAP and $\mathrm{rSO}_{2}$ values, and that the baseline $\mathrm{rSO}_{2}$ value was $75.89 \%$ in our study population. The MAP values decreased in both groups immediately after induction of anesthesia (T1), but the value of $\mathrm{rSO}_{2}$ did not show a corresponding decline. This may be related to the inhalation of $100 \%$ oxygen during anesthesia induction. The time point that corresponded to the period of deliberate hypotension was T2 (at the beginning of the operation), T3 (10 min after the operation began), T4 (20 min after the operation began), and T5 (at the end of the operation). During deliberate hypotension, the $\mathrm{rSO}_{2}$ values were lower in the intervention group compared with the control group. When baseline MAP decreased by $\sim 30 \%$, the decrease in $\mathrm{rSO}_{2}$ from the baseline was only $\sim 5 \%$ in the intervention group. In the control group, when the baseline MAP decreased as a result of the use of anesthesia, no additional decrease in $\mathrm{rSO}_{2}$ value was found. Since the reduction from baseline MAP did not proceed beyond the range of cerebral autoregulation. Both $\mathrm{MAP}$ and $\mathrm{rSO}_{2}$ values were restored to baseline levels $15 \mathrm{~min}$ after the end of the operation.

Several anesthetics and vasoactive agents have been used to establish controlled hypotension. In our study, the combined use of nicardipine and esmolol decreased the MAP to $70 \%$ of the baseline and maintained it at this level. Nicardipine is a calcium channel antagonist that induces hypotension and exerts a protective effect on renal function (22). It is also known to cause tachycardia (22) which increases myocardial metabolism and shortens diastole, thereby reducing myocardial perfusion. Esmolol is a $\beta$-blocker that can inhibit reflex tachycardia, decrease myocardial oxygen consumption, reduce cardiac output (CO), and finally lower the MAP (23). Sun et al. demonstrated that deliberate hypotension using nicardipine combined with esmolol not only maintained hemodynamic stability and decreased perioperative stress response, but also reduced the incidence of POCD in elderly patients (24). Therefore, in our study, continuous infusion of nicardipine plus esmolol was used to maintain MAP at $70 \%$ of the baseline in the intervention group. Our investigation showed that HR was relatively stable during deliberate hypotension using nicardipine plus esmolol, and that there was no significant difference between the control and intervention groups. Nicardipine plus esmolol may be a better choice to induce deliberate hypotension during FESS.

During deliberate hypotension, the reduction of MAP will lead to cerebral hypoperfusion and hypoxia, and postoperative neurological injury. In our study, we analyzed the postoperative cognitive function and did not observe any decline in either group. Furthermore, none of the patients experienced CDEs when the baseline MAP was decreased by $30 \%$. Studies have shown that patients undergoing FESS with deliberate hypotension $(>75 \%, 65-75 \%$, and $<65 \%$ of baseline) seemed to be equally safe for the patient as anesthetic management in normotension, simultaneously decreasing the complication rate, such as renal, lung, cardiovascular system damage, and postoperative cognitive decline (25).

This study has some limitations. First, we only analyzed the cognitive function on the first day postsurgery, do not evaluate the next longer period of cognitive function and other postoperative neurological injury, such as stroke and POD. Second, the level of biomarkers of cerebral ischemic change, such as S-100ß and glial fibrillary acidic protein were also not tested. Third, postoperative adverse reactions, such as nausea and vomiting were not compared between the two groups of patients.

\section{CONCLUSION}

A decrease in MAP up to a certain level will decrease $\mathrm{rSO}_{2}$ in patients undergoing FESS under general anesthesia. Our results showed that when the baseline MAP was reduced by $30 \%$, the decrease in $\mathrm{rSO}_{2}$ from the baseline was only $\sim 5 \%$, which is still within safe levels. The reduction in $\mathrm{rSO}_{2}$ is particularly associated with the decrease of MAP. Based on our findings, we recommend that the deliberate hypotensive target indicated by MAP be reduced by $30 \%$, while $\mathrm{PetCO}_{2}$ be maintained at $35-40 \mathrm{mmHg}$ and $\mathrm{HR}$ be maintained at about 60 beats per minute during FESS, which not only decreases intraoperative bleeding, improves the quality of the surgical field and reduces the duration of surgery during FESS, but also avoids the occurrence of CDEs. Therefore, nicardipine plus esmolol may be a better choice to induce deliberate hypotension during FESS. 


\section{DATA AVAILABILITY STATEMENT}

The original contributions presented in the study are included in the article/supplementary material, further inquiries can be directed to the corresponding author/s.

\section{ETHICS STATEMENT}

The studies involving human participants were reviewed and approved by Hangzhou First People's Hospital Affiliated to Zhejiang University school of Medicine. The patients/participants provided their written informed consent to participate in this study.

\section{AUTHOR CONTRIBUTIONS}

LZ and JS: conception and design. JS: administrative support and randomization and maintaining the group assignment numbers. YY and WL: anesthesia and deliberate hypotension. JX and

\section{REFERENCES}

1. Ha TN, van Renen RG, Ludbrook GL, Valentine R, Ou J, Wormald PJ. The relationship between hypotension, cerebral flow, and the surgical field during endoscopic sinus surgery. Laryngoscope. (2014) 124:222430. doi: 10.1002/lary.24664

2. Farzanegan B, Eraghi MG, Abdollahi S, Ghorbani J, Khalili A, Moshari R, et al. Evaluation of cerebral oxygen saturation during hypotensive anesthesia in functional endoscopic sinus surgery. J Anaesthesiol Clin Pharmacol. (2018) 34:503-6. doi: 10.4103/joacp.JOACP_248_17

3. Alsaleh S, Manji J, Javer A. Optimization of the surgical field in endoscopic sinus surgery: an evidence-based approach. Curr Allergy Asthma Rep. (2019) 19:8. doi: 10.1007/s11882-019-0847-5

4. Erdem AF, Kayabasoglu G, Tas Tuna A, Palabiyik O, Tomak Y, Beyaz SG. Effect of controlled hypotension on regional cerebral oxygen saturation during rhinoplasty: a prospective study. J Clin Monit Comput. (2016) 30:65560. doi: 10.1007/s10877-015-9768-6

5. Aguirre JA, Etzensperger F, Brada M, Guzzella S, Saporito A, Blumenthal S, et al. The beach chair position for shoulder surgery in intravenous general anesthesia and controlled hypotension: impact on cerebral oxygenation, cerebral blood flow and neurobehavioral outcome. J Clin Anesth. (2019) 53:40-8. doi: 10.1016/j.jclinane.2018.09.035

6. Murkin JM, Arango M. Near-infrared spectroscopy as an index of brain and tissue oxygenation. Br J Anaesth. (2009) 103(suppl. I): i3-13. doi: 10.1093/bja/aep299

7. Nielsen HB. Systematic review of near-infrared spectroscopy determined cerebral oxygenation during non-cardiac surgery. Front Physiol. (2014) 5:93. doi: 10.3389/fphys.2014.00093

8. Heller JA, DeMaria Jr S, Govindaraj S, Lin HM, Fischer GW, et al. Cerebral oximetry monitoring during sinus endoscopy. Laryngoscope. (2015) 125:E127-31. doi: 10.1002/lary.25027

9. Yang W, Wang G, Li H, Yan X, Ren Y, Wang Y, et al. The 15 degrees reverse trendelenburg position can improve visualization without impacting cerebral oxygenation in endoscopic sinus surgery-A prospective, randomized study. Int Forum Allergy Rhinol. (2021) 11:993-1000. doi: 10.1002/alr. 22734

10. Saxena A, Nekhendzy V. Anesthetic considerations for functional endoscopic sinus surgery. J Head Neck Anesth. (2020) 4:e25. doi: 10.1097/HN9.0000000000000025

11. $\mathrm{Yu} \mathrm{Q}$, Qi J, Wang Y. Intraoperative hypotension and neurological outcomes. Curr Opin Anaesthesiol. (2020) 33:64650. doi: 10.1097/ACO.0000000000000904

12. Sun LY, Chung AM, Farkouh ME, van Diepen S, Weinberger J, Bourke $\mathrm{M}$, et al. Defining an intraoperative hypotension threshold
YH: collection of data. YL: fromme score. LZ: data analysis. All authors wrote and approved the manuscript.

\section{FUNDING}

The project was supported by Zhejiang University of Traditional Chinese Medicine Postgraduate Top-notch Innovative Talent Cultivation Program (ZHYD2017-17), The Key Project of Joint Pre-research Fund (YYJJ2019Z03) for Clinical Scientific Research of Hangzhou First People's Hospital Affiliated to Zhejiang University, Medical Scientific Research Foundation of Zhejiang province (2015ZYC-A34), and the Natural Science Foundation of Zhejiang Province (LY21H090005).

\section{ACKNOWLEDGMENTS}

We are very grateful to Guoming Xie, MD who provided modifications to this paper.

in association with stroke in cardiac surgery. Anesthesiology.

(2018) 129:440-7. doi: 10.1097/ALN.0000000000002298

13. Bijker JB, Persoon S, Peelen LM, Moons KG, Kalkman CJ, Kappelle LJ, et al. Intraoperative hypotension and perioperative ischemic stroke after general surgery: a nested case-control study. Anesthesiology. (2012) 116:65864. doi: 10.1097/ALN.0b013e3182472320

14. Maheshwari K, Ahuja S, Khanna AK, Mao G, Perez-Protto S, Farag E, et al. Association between perioperative hypotension and delirium in postoperative critically ill patients: a retrospective cohort analysis. Anesth Analg. (2020) 130:636-43. doi: 10.1213/ANE.0000000000004517

15. Meng L, Wang Y, Zhang L, McDonagh DL. Heterogeneity and variability in pressure autoregulation of organ blood flow: lessons learned over $100+$ years. Crit Care Med. (2019) 47:436-48. doi: 10.1097/CCM.0000000000003569

16. Huber W, Zanner R, Schneider G, Schmid R, Lahmer T. Assessment of regional perfusion and organ function: less and non-invasive techniques. Front Med. (2019) 6:50. doi: 10.3389/fmed.2019.00050

17. Kim J, Shim JK, Song JW, Kim EK, Kwak YL. Postoperative cognitive dysfunction and the change of regional cerebral oxygen saturation in elderly patients undergoing spinal surgery. Anesth Analg. (2016) 123:43644. doi: 10.1213/ANE.0000000000001352

18. Liu Y, Chen K, Mei W. Neurological complications after cardiac surgery: anesthetic considerations based on outcome evidence. Curr Opin Anaesthesiol. (2019) 32:563-7. doi: 10.1097/ACO.0000000000000755

19. Wang X, Feng K, Liu H, Liu Y, Ye M, Zhao G, et al. Regional cerebral oxygen saturation and postoperative delirium in endovascular surgery: a prospective cohort study. Trials. (2019) 20:504. doi: 10.1186/s13063-019-3586-y

20. Picton P, Dering A, Alexander A, Neff M, Miller BS, Shanks A, et al Influence of ventilation strategies and anesthetic techniques on regional cerebral oximetry in the beach chair position: a prospective interventional study with a randomized comparison of two anesthetics. Anesthesiology. (2015) 123:765-74. doi: 10.1097/ALN.0000000000000798

21. Lee JH, Min KT, Chun YM, Kim EJ, Choi SH. Effects of beach-chair position and induced hypotension on cerebral oxygen saturation in patients undergoing arthroscopic shoulder surgery. Arthroscopy. (2011) 27:88994. doi: 10.1016/j.arthro.2011.02.027

22. Kim JE, Lee JS, Kim MK, Kim SH, Kim JY. Nicardipine infusion for hypotensive anesthesia during orthognathic surgery has protective effect on renal function. J Oral Maxillofac Surg. (2014) 72:41-6. doi: 10.1016/j.joms.2013.08.011

23. Das A, Chhaule S, Bhattacharya S, Basunia S, Mitra T, Halder P, et al. Controlled hypotension in day care functional endoscopic sinus surgery: a comparison between esmolol and dexmedetomidine: a prospective, double-blind, randomized study. Saudi J Anaesth. (2016) 10:276-82. doi: 10.4103/1658-354X.174919 
24. Sun SH, Yang L, Sun DF, Wu Y, Han J, Liu RC, et al. Effects of vasodilator and esmolol-induced hemodynamic stability on early post-operative cognitive dysfunction in elderly patients: a randomized trial. Afr Health Sci. (2016) 16:1056-66. doi: 10.4314/ahs.v1 $6 \mathrm{i} 4.23$

25. Nowak S, Oldak A, Kluzik A, Drobnik L. Impact of controlled induced hypotension on cognitive functions of patients undergoing functional endoscopic sinus surgery. Med Sci Monitor. (2016) 22:898-907. doi: 10.12659/MSM.895964

Conflict of Interest: The authors declare that the research was conducted in the absence of any commercial or financial relationships that could be construed as a potential conflict of interest.
Publisher's Note: All claims expressed in this article are solely those of the authors and do not necessarily represent those of their affiliated organizations, or those of the publisher, the editors and the reviewers. Any product that may be evaluated in this article, or claim that may be made by its manufacturer, is not guaranteed or endorsed by the publisher.

Copyright (c) 2021 Zhang, Yu, Xue, Lei, Huang, Li and Sun. This is an open-access article distributed under the terms of the Creative Commons Attribution License (CC BY). The use, distribution or reproduction in other forums is permitted, provided the original author(s) and the copyright owner(s) are credited and that the original publication in this journal is cited, in accordance with accepted academic practice. No use, distribution or reproduction is permitted which does not comply with these terms. 